\title{
Performance Analysis of the ATLAS Second-Level Trigger Software
}

\author{
J. A. C. Bogaerts, David R. Botterill, Weidong Li, R. P. Middleton, Per Werner, F. J. Wickens, and Haimo Zobernig
}

\begin{abstract}
In this paper, we analyze the performance of the prototype software developed for the ATLAS Second-Level Trigger. The $\mathrm{OO}$ framework written in $\mathrm{C}++$ has been used to implement a distributed system which collects (simulated) detector data on which it executes event selection algorithms. The software has been used on testbeds of up to 100 nodes with various interconnect technologies. The final system will have to sustain traffic of $\sim 40 \mathrm{~Gb} / \mathrm{s}$ and require an estimated number of $\sim 750$ processors. Timing measurements are crucial for issues such as trigger decision latency, assessment of required CPU and network capacity, scalability, and load-balancing. In addition, final architectural and technological choices, code optimization, and system tuning require a detailed understanding of both CPU utilization and trigger decision latency. In this paper, we describe the instrumentation used to disentangle effects due to such factors as OS system intervention, blocking on interlocks (applications are multithreaded), multiple CPUs, and I/O. This is followed by an analysis of the measurements and concluding with suggestions for improvements to the ATLAS Trigger/DAQ dataflow components in the next phase of the project.
\end{abstract}

Index Terms-Computer network performance, data acquisition, data communication, high-energy physics, timing, trigger.

\section{INTRODUCTION}

A TLAS $^{1}$ is a general-purpose detector designed to study proton-proton collisions at the Large Hadron Collider at the European Center for Nuclear Research (CERN). Bunches of protons running in opposite directions around the accelerator ring will cross in the center of ATLAS at a frequency of $40 \mathrm{MHz}$ (every $25 \mathrm{~ns}$ ) and at each crossing several collisions will occur. Given the large number of electronic channels, the expected rate of data production is of the order of $10^{1 \tilde{5}} \mathrm{~B} / \mathrm{s}$. A three-level selection (trigger) system will be used to filter these data to reduce them to a more manageable size for long-term storage [1].

Events which pass the First-Level Trigger are stored in approximately 1500 read-out buffers (RoBs). In addition, the First-Level Trigger will produce region of interest (RoI) pointers, indicating the most significant activity in the detector. The Second-Level Trigger will use data from the RoIs to reduce

Manuscript received June 4, 2001; revised October 22, 2001.

J. A. C. Bogaerts and P. Werner are with the European Center for Nuclear Research (CERN), 1211 Geneva 23, Switzerland (e-mail: Joannes.Andreas.Bogaerts@cern.ch; Per.Werner@cern.ch).

D. R. Botterill, W. Li, R. P. Middleton, and F. J. Wickens are with the Rutherford Appleton Laboratory, Oxon OX11 0QX, U.K. (e-mail: David.Botterill@rl.ac.uk; Weidong.Li@rl.ac.uk; R.P.Middleton@rl.ac.uk; F.J.Wickens@rl.ac.uk).

H. Zobernig is with the Department of Physics, University of Wisconsin, Madison, WI 53706 USA (e-mail: Haimo.Zobernig@ cern.ch).

Publisher Item Identifier S 0018-9499(02)03926-6.

${ }^{1}$ Available: http://atlasinfo.cern.ch/Atlas/Welcome.html the rate from approximately 100 to $1 \mathrm{kHz}$. It is estimated that the Second-Level Trigger will require a farm of some 750 LVL2 Processors. A LVL2 Supervisor interfaces to the First-Level Trigger and provides the RoI pointers. A network, which in the final system will have to sustain traffic of $\sim 40 \mathrm{~Gb} / \mathrm{s}$, interconnects RoBs, LVL2 Processors, and LVL2 Supervisor. The third stage of event selection, with a further reduction factor of $\sim 10$, is provided by the Event Filter using the full detector data after event building.

The Reference Software ${ }^{2}$ is a prototype implementation to study the ATLAS Second-Level Trigger. Applying OO techniques, it was written in $\mathrm{C}++$ and used to implement a distributed system to collect (simulated) detector data and execute event selection algorithms. The software has been used on testbeds of up to 100 nodes to investigate various interconnect technologies [1]. Trigger and data rates have been measured either with dummy data or simulated data and algorithms [1] accompanied by simulations to study scaling to realistic system sizes [1]. In this paper, we describe detailed internal timing measurements made on small (3-6 node) systems. The paper includes a description of the instrumentation of the software and tools used, together with some results. We conclude with some lessons to be carried forward to the next phase of the ATLAS Trigger/DAQ project.

\section{A. Motivation}

Previous tests of the Reference Software concentrated on measurements of trigger rates and data volumes as a function of parameters such as: system size (RoBs and LVL2 Processors), RoB data sizes, inclusion of algorithms, networking technologies, message passing protocols and usage of mono or multi-CPU based processors. Results thus obtained are difficult to analyze, blind to mis-synchronization, and give little input to system modeling. We, therefore, decided to instrument the code for more detailed timing measurements using a small system consisting of one Supervisor, one LVL2 Processor and one or a few RoBs. The aim was to profile the software, to separate time spent in communication from other processing and idle time introduced by interlocks and I/O (applications are multithreaded) and to investigate the effectiveness of multi-CPU processors. This will help the design of the next prototype and provide more detailed parameters for modeling.

\section{B. Methods and Tools}

Three complementary approaches have been used to evaluate the performance.

\footnotetext{
${ }^{2}$ ATLAS Second-Level Trigger Pilot Project Reference Software. [Online] Available: http://atlas.web.cern.ch/Atlas/project/LVL2testbed/www/
} 


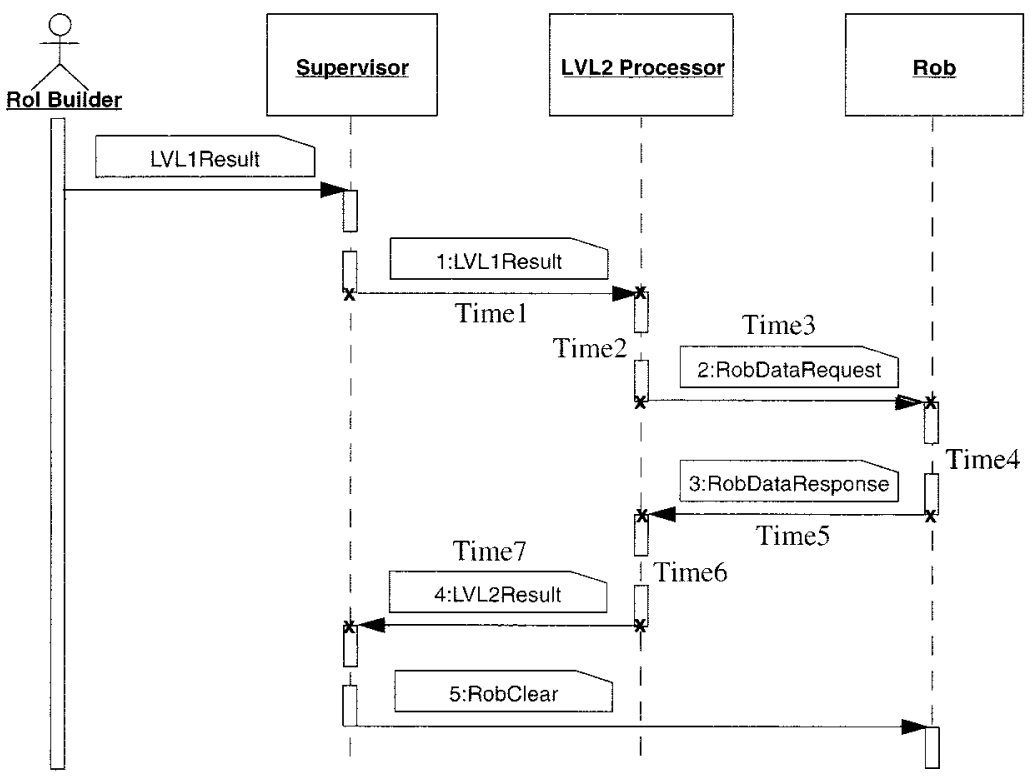

Fig. 1. Sequence diagram showing the exchange of messages between Supervisor, LVL2 Processor, and RoB.

TAU [2] is a tool that supports automatic instrumentation, data collection, analysis, profiling and tracing of complex systems consisting of multiple nodes with multithreaded applications implemented in C++. TAU works in many environments, including Intel Linux clusters, templated GNU C++ and pthreads. It is particular useful to provide an overview of which part of the code is most frequently used and give indications where most of the time is spent.

NetLogger ${ }^{3}$ provides mechanisms to generate time-stamps at selected places in code running in a distributed system, plus tools to visualise the results. Manually inserted macros are used to generate the time-stamps, which are written to disk files for later analysis. This method is particularly useful for inter-node measurements.

Finally, we have instrumented the code to measure and histogram time intervals using the local clock. This was based on a general system-monitoring facility, already available in the Reference Software, to collect simple quantities such as counters and histograms. Since information in histograms cannot be correlated afterwards, this method is only suitable for intra-node measurements. However, it does not require synchronised clocks nor add much to the execution time.

\section{System OVERVIEW}

The functionality of the Second-Level Trigger as implemented in the Reference Software requires three applications each running on separate nodes: Supervisor, LVL2 Processor, and RoB. A full configuration includes setting various parameters, such as the number of event descriptors each LVL2 processor is allowed to buffer, number of LVL2 Processors, number of Worker threads for each processor, execution time of (dummy) algorithms, number of RoBs to collect RoI data from, mapping of RoBs on the detector readout, and RoI data size.

${ }^{3}$ A Methodology for Monitoring and Analysis of Distributed Systems. [Online]. Available: http://www-didc.lbl.gov/NetLogger
The operation of the system is illustrated by the UML sequence diagrams of Figs. 1 and 2. The event processing is initiated by the Supervisor sending a LVL1Result to the LVL2 Processor where it is received by an Input Thread and despatched to a Worker Thread. Based on the RoI pointers contained in the LVL1Result, the Worker Thread will send requests for data to the appropriate RoBs and wait for a response. Again, the Input Thread is responsible for receiving the RoB data and despatching it to the appropriate Worker Thread which, upon re-activation, processes the RoB data to finally return the trigger decision as a LVL2Result back to the Supervisor. All applications follow the same pattern for communication: messages are sent directly by a Worker Thread, but all data is received by an Input Thread, which despatches data to the appropriate Worker Thread using blocking get/put operations on shared queues (in practice, only get operations will block). This simplifies the instrumentation of the code. Typically, timing measurements are inserted before and after all send/receive and all get/put operations. This is sufficient to measure time spent in communication, as well as waiting time, which only occurs in receive-and-get operations.

\section{Software Profiling With TAU}

We used the ability of the TAU [2] profiler to equip every function and method call in a $\mathrm{C}++$ program to measure the time elapsed between the entry into the function and the return from it. TAU was chosen because it supports profiling of dynamically loaded shared libraries, handles multithreaded programs, and is readily available as free software. In addition to its normal mode, where functions are instrumented manually by inserting certain macro calls at strategically chosen places, TAU has a mode of operation which allows the entire Reference Software to be instrumented automatically. We used this mode, since our main goal using TAU was to get an understanding of where time is spent at the function level and to discover any unexpected large time consumers. 


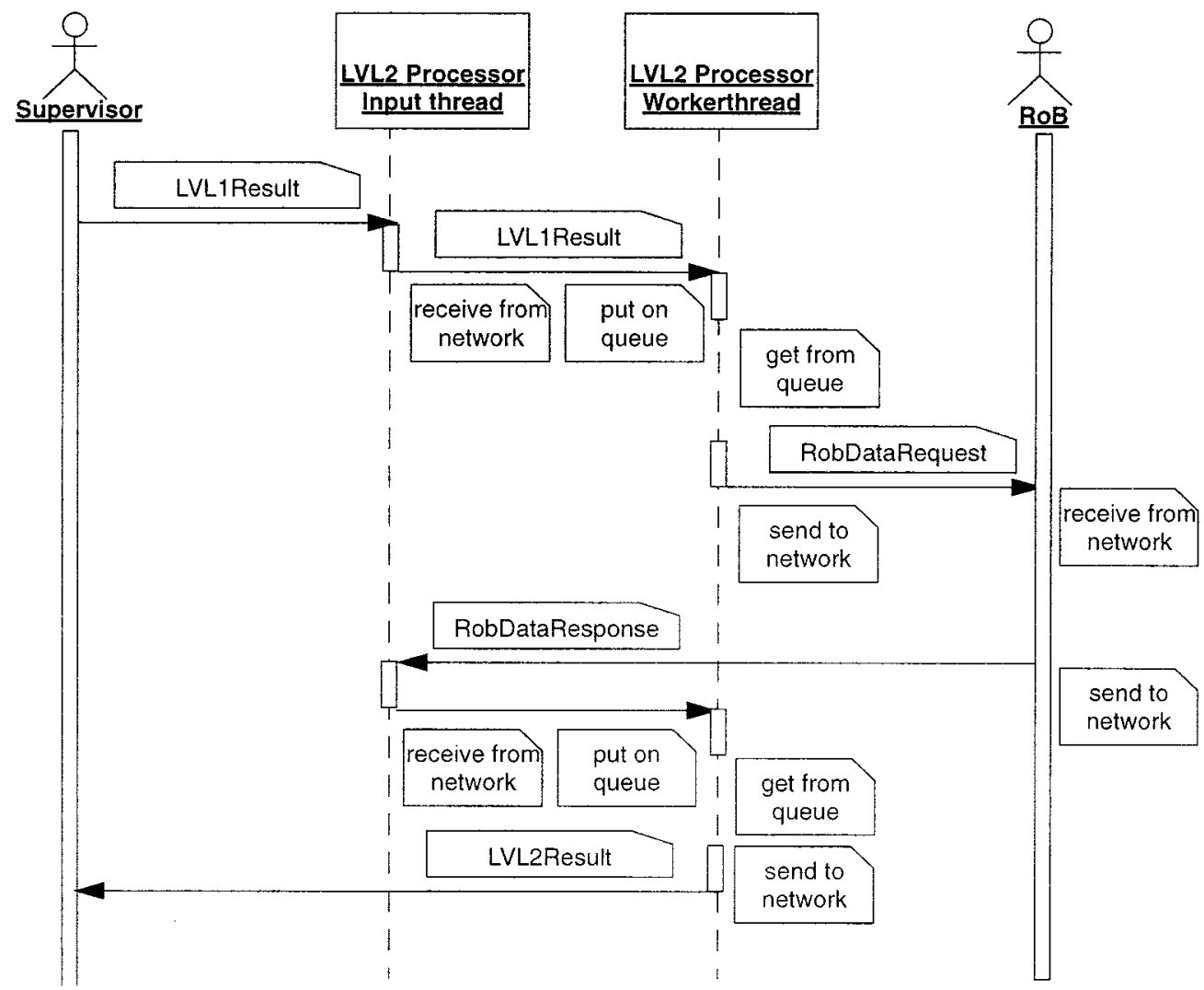

Fig. 2. Sequence diagram showing the internal communication between Input and Worker Thread in the LVL2 Processor.

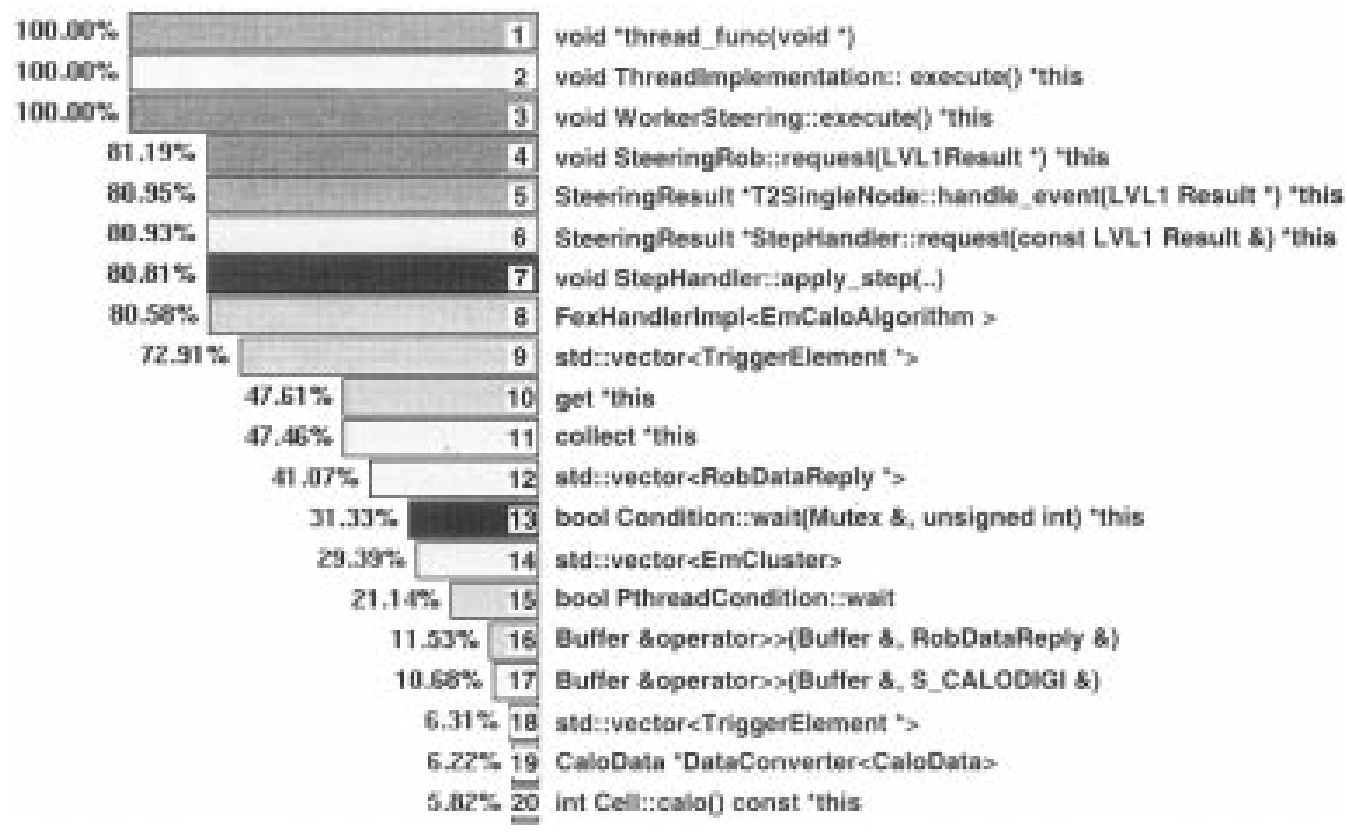

Fig. 3. Inclusive fraction (in percent) of the total running time, counting the time spent in each function and all others it calls. Only the 20 largest time consumers are shown. Some function signatures are truncated.

Fig. 3 illustrates a typical runtime profile of one Worker thread on the LVL2 processor, using simulated events that trigger in LVL1. This run was made with one of each Supervisor, RoB and LVL2 Processor, using one Worker thread and one CPU on each of the three nodes. TCP/IP over $100 \mathrm{Mb} / \mathrm{s}$
Ethernet was used for communication. Real trigger algorithms were employed, resulting in a rejection rate of about $40: 1$ events.

Lines 1-3 are the thread's work function, hence (close to) $100 \%$. Line 4 shows that $\sim 80 \%$ of the time is spent waiting for 
and processing RoB data. Most of that is spent in code handling the trigger algorithms (lines 5-9). Approximately 30\% is waiting time for RoB data (lines 13,15 ), while $~ 50 \%$ is actual Trigger processing (lines 10-11). Lines 14 and 16-20 show some of the larger time consumers, among which happen to be calorimeter feature extraction and some stream input operators used for data unpacking. These will need careful optimization in future implementations.

\section{NetLogger Measurements of Communication TIMING}

From the time that an event is allocated to a given LVL2 processor until the LVL2 Result is returned, various messages must pass either between the nodes involved (Supervisor, LVL2 Processor, RoBs) or between the Input thread and the appropriate Worker thread in these nodes. NetLogger has been used to generate time-stamps as each message leaves the source thread or is received in the destination thread. The time for messages between nodes is mainly determined by the network hardware and software (which may include internal buffering), but will also be affected by the scheduling of the source thread or the Input thread in the destination node. In contrast, the Input and Worker threads communicate via shared queues and consequently the message latency will be determined by the length of the queue in the node and the scheduling of the two threads. Analysis of the time-stamps allows typical message passing delays to be determined and deviations due to scheduling or network problems to be seen.

\section{A. Instrumentation}

NetLogger is normally used with NTP to give a precision between nodes of $\sim 250 \mu \mathrm{s}$. However, this is comparable to the message passing delays to be measured. Greater precision was achieved by using the Parallel Port Timing system from the Weizmann Institute of Science, Tel Aviv, Israel. This system provides a global clock ( 1 tick $=250 \mathrm{~ns}$ ) to the parallel port of each processor node. Additional software synchronised the local clock on each node and maintained synchronization to typically better than $5 \mu \mathrm{s}$, with adjustments every $5 \mathrm{~s}$. To avoid problems in this multithreaded environment, NetLogger was used in a thread-safe mode with each thread writing to a separate memory buffer. Data was written to disk at the end of each run. Each time-stamp recorded the identification of the call, plus the event number and the time. It was found that each NetLogger call adds $\sim 30 \mu$ s to the execution time. After the run, the time-stamps were analyzed by histogramming differences between time-stamps and using the NetLogger visualization tool.

\section{B. Measurements}

Measurements were made using $450-\mathrm{MHz}$ dual-processor PCs, running Red Hat Linux 6.1 and using TCP/IP over 100 $\mathrm{Mb} / \mathrm{s}$ Ethernet. Each configuration used a single Supervisor node, with a single LVL2 Processor collecting data from 1-3 RoBs. The data size collected from each RoB was varied from
TABLE I

TIME INTERVALS FOR VARIOUS ROB DATA SIZES

\begin{tabular}{l|c|c|c|c|c|c}
\hline Data size & 64 & $1 \mathrm{~K}$ & $4 \mathrm{~K}$ & $8 \mathrm{~K}$ & $\mathrm{t} 0$ & $\delta \mathrm{t}$ \\
\hline Interval & $\mu \mathrm{s}$ & $\mu \mathrm{s}$ & $\mu \mathrm{s}$ & $\mu \mathrm{s}$ & $\mu \mathrm{s}$ & $\mu \mathrm{s} / \mathrm{KB}$ \\
\hline Time1 & 183 & 182 & 182 & 182 & 182 & 0 \\
\hline Time2 & 179 & 179 & 181 & 178 & 180 & 0 \\
\hline Time3 & 171 & 176 & 168 & 166 & 174 & 0 \\
\hline Time4 & 166 & 191 & 257 & 354 & 165 & 23 \\
\hline Time5 & 185 & 359 & 607 & 955 & 223 & 91 \\
\hline Time6 & 187 & 211 & 274 & 364 & 186 & 22 \\
\hline Time7 & 172 & 174 & 176 & 171 & 174 & 0 \\
\hline
\end{tabular}

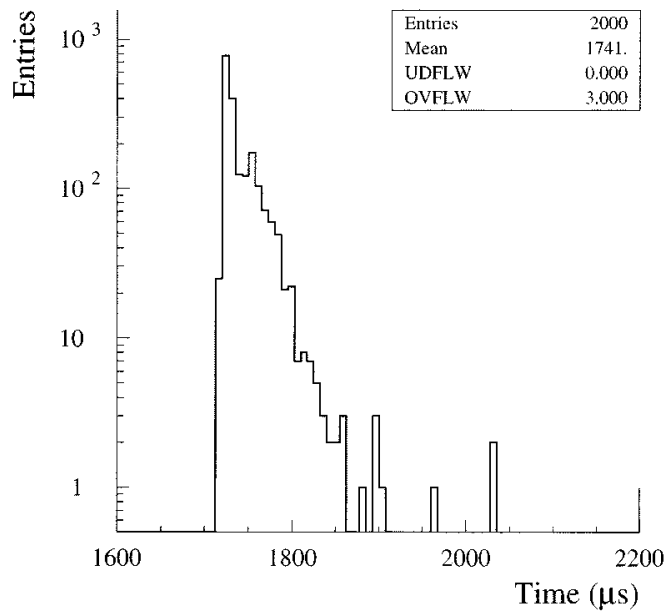

Fig. 4. Distribution of time measured in the LVL2 Processor for collecting 4 $\mathrm{kB}$ of data from each of three RoBs.

$64 \mathrm{~B}$ to $8 \mathrm{kB}$. No algorithm processing time was included in the Worker threads.

To determine the basic message-passing times, a configuration was run with a single RoB, a single Worker thread, and only one event allowed in the system at a time, all computers running in single processor mode. Seven time intervals (Time1-Time7) were measured, as shown in Fig. 1. The results are shown in Table I, where the final two columns show the results of straight-line fits for the dependence on RoB data size. It can be seen that Time5, which includes the transmission of the RoB data across the network, has a large dependence. Time4 and Time6, which include memory copies of the RoB data in the RoB and LVL2 processor, respectively, have a modest dependence. The variation in the time intervals is generally small. Fig. 4 shows the distribution of times for collecting $4 \mathrm{kB}$ of data from each of three RoBs.

The NetLogger Visualization tool was used to study the lifeline of each event as it passes through the system. With a wellbalanced system, events proceed in a uniform manner through their processing. However, when a resource is under pressure the flow of events is less smooth. Thus, in Fig. 5, with four Worker threads competing against each other (and the Input thread), some events take many milliseconds to complete receipt of the received RoB data, as their thread fails to be scheduled. Similar disruption to the flow of events has been seen when the network resources are under extreme pressure. 


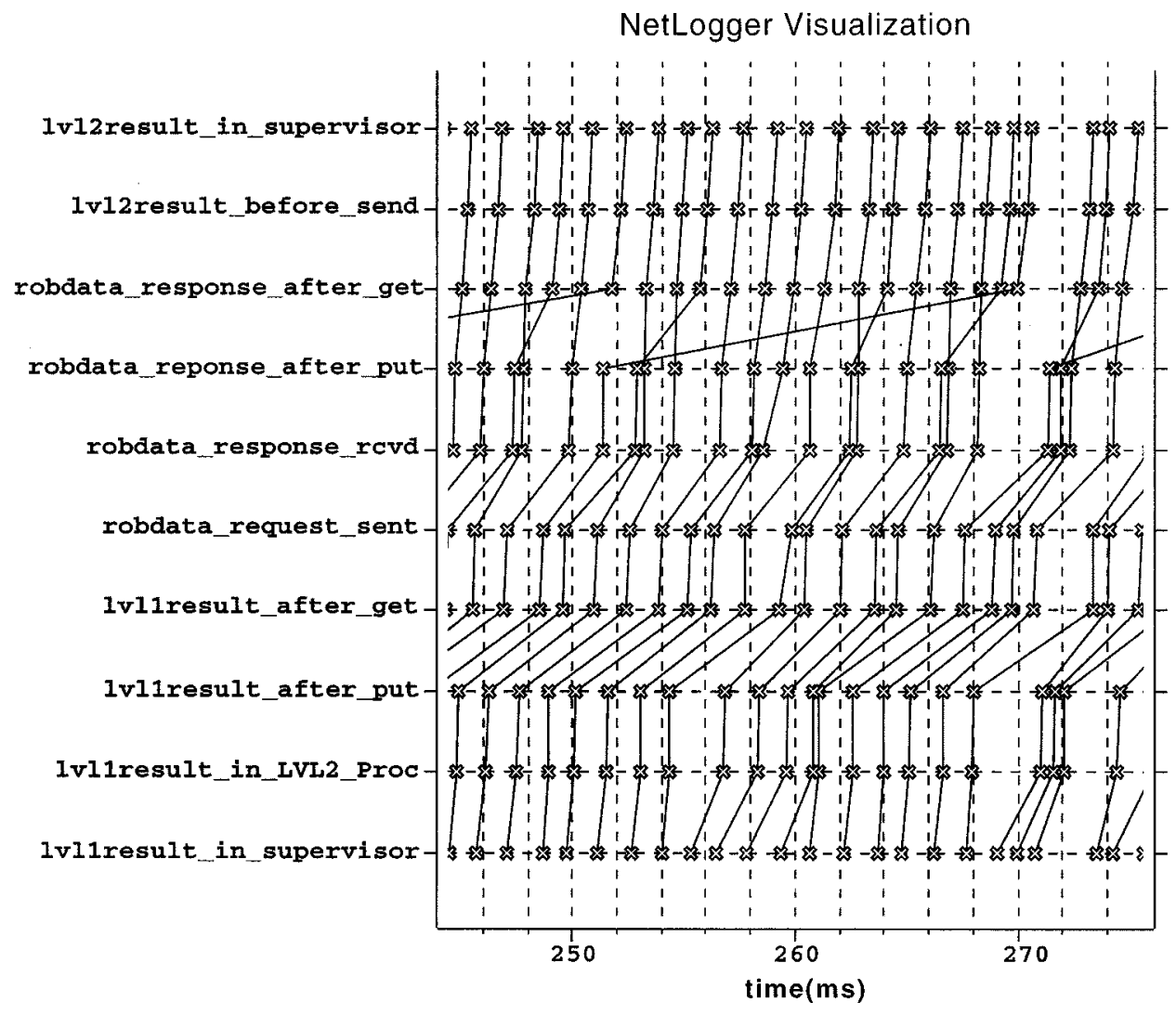

Fig. 5. Netlogger Visualization of a LVL2 Processor with four Worker Threads.

\section{MEASUREMENTS OF CPU UtILIZATION}

The multithreaded approach lends itself to the use of symmetric multiprocessor (SMP) systems. The optimum number of threads obviously depends on the ratio of execution to waiting time, and on the number of CPUs. In Sections V-A-C, we describe measurements made on mono and multiprocessor systems to assess the achieved CPU utilization for various load conditions.

\section{A. Instrumentation}

The LVL2 Processor has been instrumented to measure the amount of processing time required for each event. The general architecture of the LVL2 Processor is illustrated by the sequence diagram of Fig. 2. There is an Input thread that receives all inputs: the LVL1 Results from the supervisor and the RoB Data Responses from the RoBs after issuing a RoB Data Request. Data from the Supervisor and RoBs are dispatched to different shared queues. Computations are carried out in Worker threads, each looking after an entire event. The LVL1 result is obtained from the Supervisor queue on a first-come, first-serve basis.

\section{B. Measurements}

A series of measurements has been carried out on a system of one Supervisor, one LVL2 Processor, and one RoB running Red Hat Linux version 6.1 on PCs of $450 \mathrm{MHz}$ booted in single CPU mode and interconnected through a $100 \mathrm{Mb} / \mathrm{s}$ Ethernet switch. To investigate the impact of multiple CPUs, the LVL2 Processor was also booted in dual CPU mode and even replaced by a 550-MHz quad CPU processor. TCP/IP was used for the message passing; it has been verified that the Supervisor saturated the LVL2 Processor; 1024 B of data were requested from one RoB for each event; the number of Worker threads was varied from 1 to 16; the CPU load was varied between $0,0.5,1$, and 2 $\mathrm{ms}$; the number of CPUs was 1,2, or 4 . In all cases, the event rate and CPU utilization was measured. The waiting time to receive $1024 \mathrm{~B}$ of data was typically $\sim 500 \mu \mathrm{s}$. The code was instrumented to measure the total execution time of each Worker, as well as the waiting time to receive RoB data. The difference of the two gives the CPU time used by each Worker thread (CPU time as measured by the OS is not reliable for these small intervals). Similarly, the execution time of the Input thread, typically $\sim 20 \mu$ s, was measured for each event. The Real Time clock with a resolution of a few microseconds was used and the measured time intervals were histogrammed on the fly. For many measurements, the I/O system and CPU capacity were driven to their limits. Consequently, threads were frequently pre-empted and waiting times appeared longer because the CPU was busy. However, for a small number of threads, histograms showed more prominent peaks at the minimum value of the distribution, thus providing consistent measurements for the true time intervals. Time spent in the OS, such as context switches but, more importantly, also protocol processing for TCP/IP or UDP, have not been measured. The latter, which may use up a considerable fraction of the available CPU time and limit the data rate for smaller data sizes in cases where I/O dominates the processing, has been extrapolated from measurements ( $\sim 200 \mu$ s per event). 


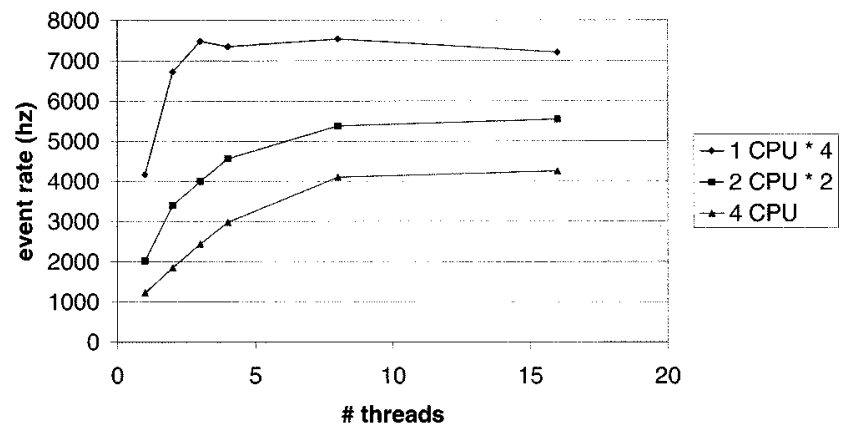

Fig. 6. Event (trigger) rate as a function of the number LVL2 Processor Worker threads with no algorithms running. The plot compares the rate of a single quad-CPU with the rate obtained by two dual or four mono-CPU processors. The system is clearly $\mathrm{I} / \mathrm{O}$ bound.

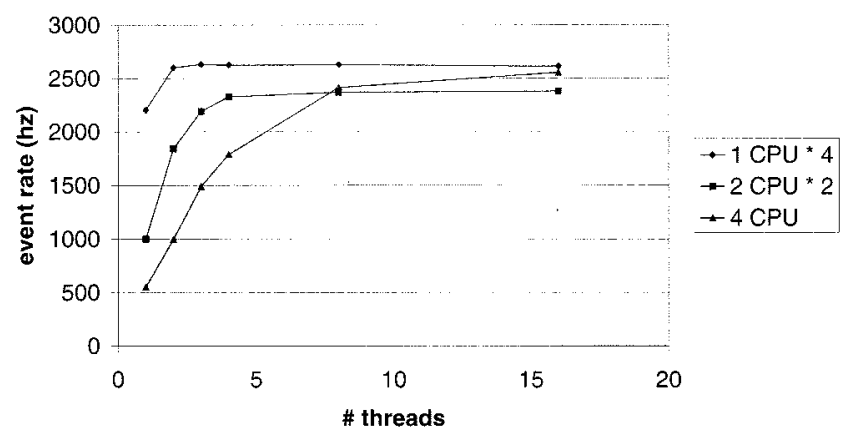

Fig. 7. As Fig. 5, but with an artificial CPU load of $1 \mathrm{~ms}$, representing time spent in algorithms. SMPs clearly become as efficient as monoprocessors when the system becomes CPU bound.

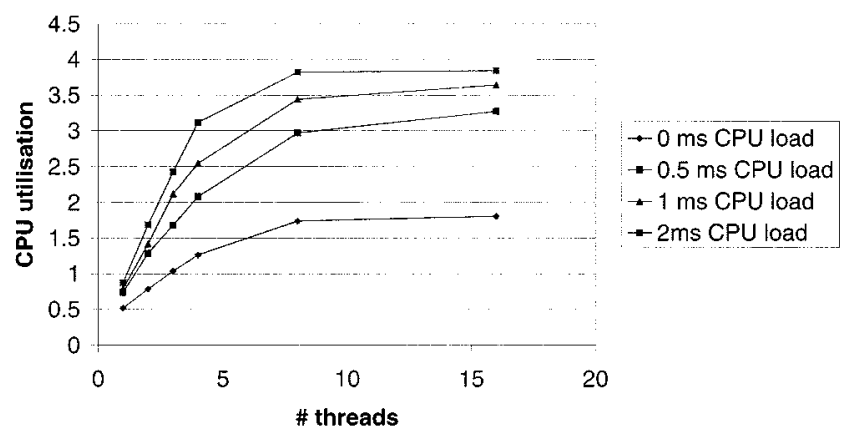

Fig. 8. Plot showing the CPU usage as a function of the number of threads with an additional CPU load of varying between 0 and $2 \mathrm{~ms}$ per event for a quad-CPU processor. With no CPU load, nearly two CPUs are used for communication and framework overheads; nearly all four CPUs are used, with an additional load of 2 ms per event.

The results are summarized in Figs. 6-8. To allow a better comparison between mono and multiprocessors the event rate has been multiplied by a factor of 2 and 4 for a dual and single CPU machine.

\section{Discussion}

All plots show the characteristic rise of the event rate to a maximum value as the number of Worker threads increases. As expected, more threads are needed to reach the plateau when I/O dominates with a higher ratio of waiting versus execution time. Similarly and trivially, the number of threads should exceed the number of CPUs. When processing dominates the event rate scales with the number of CPUs. When I/O dominates, four monoprocessors, or at least four independent network interfaces and switch connections, clearly perform better, though in this measurement the quad-processor is somewhat disfavored by the much higher RoB access rate.

\section{SUMMARY AND CONCLUSION}

We have demonstrated three ways of instrumenting multinode software for timing measurements. Manual insertion of code for timing measurements using macros (which may be compiled out) is perfectly feasible and provides much useful information. This should be pursued in future prototypes.

Profiling with TAU results in large amounts of data that need to be well understood. The present exercise provided the machinery needed to profile future versions of the software. It is hoped that profiling can be made a standard tool for our developers.

NetLogger was particularly useful to understand the flow of events between threads and processors, and for checking correlations between the measured time-stamps. Additional clock synchronization hardware and software allowed a precision of a few microseconds to be obtained. For most normal configurations, the flow of events through the Reference Software was found to be free of problems.

The multithreaded implementation of, in particular, the LVL2 Processor with single Input and multiple Worker threads functions well, does not lead to large overheads, and allows efficient CPU utilization of both mono- and multi-CPU systems. The latter provided CPU power that scales well to large systems, provided that enough $\mathrm{I} / \mathrm{O}$ capacity is available.

\section{ACKNOWLEDGMENT}

The authors would like to thank the many members of the ATLAS Trigger/DAQ community for their contributions who made this work possible. Special thanks are due to the authors of the Reference Software, without whom there would have been nothing to measure. They would also like to thank L. Levinson, Weizmann Institute of Science, and M. Dobson, CERN, for providing hardware and software for clock synchronization.

\section{REFERENCES}

[1] ATLAS, "ATLAS High-Level Triggers, DAQ and DCS," ATLAS Collaboration, CERN/LHCC/2000-17, 2000

[2] TAU. (1999) Tuning and Analysis Utilities. Dept. of Comput. Info. Sci., Univ. of Oregon, Corvallis, OR. [Online] Available: http://www.acl.lanl.gov/tau 